\title{
Application of Van der Pol oscillator screening for peripheral arterial disease in patients with diabetes mellitus
}

\author{
Jianxing $\mathrm{Wu}^{{ }^{*}}$, Chianming $\mathrm{Li}^{1,2}$, Weiling Chen ${ }^{1}$, Chiahung Lin ${ }^{3}$, Tainsong Chen ${ }^{{ }^{*}}$ \\ ${ }^{1}$ Department of Biomedical Engineering, National Cheng Kung University, Tainan City, Taiwan \\ ${ }^{2}$ Division of Infectious Diseases, Department of Medicine of Chi Mei Medical Center, Tainan City, Taiwan \\ ${ }^{3}$ Department of Electrical Engineering, Kao-Yuan University, Kaohsiung City, Taiwan \\ Email: “ian0218@gmail.com, 235813cmli@gmail.com, 1ynnchen.k@gmail.com, eech153@gmail.com, ${ }^{*}$ chents@mail.ncku.edu.tw
}

Received 7 November 2013; revised 4 December 2013; accepted 12 December 2013

Copyright (C) 2013 Jianxing Wu et al. This is an open access article distributed under the Creative Commons Attribution License, which permits unrestricted use, distribution, and reproduction in any medium, provided the original work is properly cited. In accordance of the Creative Commons Attribution License all Copyrights (C) 2013 are reserved for SCIRP and the owner of the intellectual property Jianxing Wu et al. All Copyright (C) 2013 are guarded by law and by SCIRP as a guardian.

\begin{abstract}
This paper proposes a Van der Pol (VDP) oscillator screening for peripheral arterial disease (PAD) in patients with diabetes mellitus. The long-term elevated blood sugar levels produce a high risk of peripheral neuropathy and peripheral vascular disease, especially in the foot of a diabetic. Early detection is important, in order to prevent foot problems for diabetic patients with PAD. Photoplethysmography (PPG) is a non-invasive method for the detection of blood volume changes in peripheral arteries. Because of changes in the resistance-compliance, the rise time and transit time for the PPG signals increase and change in their shape are highly correlated with PAD severity. In this study, the Burg autoregressive (AR) method is used to determine the characteristic frequencies of the right- and left-side PPG signals. For bilateral frequency spectra, the VDP oscillator uses asynchronous signals. This produces damped sinusoidal responses and the oscillation overshoot (OS) is an approximating function only of the damped factor. This index is used to estimate the degree of PAD, including normal the condition and diabetic patients with PAD. The results show that the proposed method is efficient and more accurate in the estimation of PAD.
\end{abstract}

Keywords: Van der Pol (VDP) Oscillator; Peripheral Arterial Disease (PAD); Burg Autoregressive Method; Oscillation Overshoot

${ }^{*}$ Corresponding authors

\section{INTRODUCTION}

Type 2 diabetes is a chronic, metabolic disease that is prevalent in developed and developing countries, associated with mortality and morbidity due to increasing risk factor for cardiovascular diseases. Patients with diabetes mellitus incur substantantial medical expenditure to control their glycemic concentrations and complications. Peripheral arterial disease (PAD) is highly prevalent in patients with diabetes mellitus. However, it is usually underestimated by patients as well as physicians, especially in the foot of diabetics, mostly contributing to the slow progress of arterial atherosclerosis in lower limbs. Manifestations of this peripheral arterial obstruction can vary from a symptom to total occlusion or gangrene of toe or foot [1]. Besides classic presentation of intermittent claudication, foot ulceration or diabetic foot may develop, signaling a significant degree of arterial occlusion and high risk for future amputation. PAD is responsible for $47 \%$ of amputations in diabetic patients [1]. In recent years, many imaging techniques, such as Doppler ultrasound, colour duplex ultrasound (CDU), X-ray angiography, computed tomographic angiography (CTA), and magnetic resonance angiography (MRA), have been used to diagnose PAD, verifying the presence of a stenosis inside the vasculature of diabetes [2-4]. Although these methodologies are reliable and highly accurate, a noninvasive and inexpensive technique for early diagnosis and monitoring of PAD remains unsettled in hospitals and primary care settings. Modern ultrasound technique is well-developed, compact, fast, and free of irradiation, but they can only be correctly operated by a well-trained technician clinical physician. Therefore, this study pro- 
poses a non-invasive means of measurement and an automatic diagnostic algorithm to estimate the degree of $\mathrm{PAD}$, purporting for screening and monitoring of lower limb vascular occlusion for high-risk groups like patients of diabetes, dyslipidemia, and hypertension, and the elderly.

Photoplethysmography (PPG) is an optical and noninvasive technique, widely applied to measure oxygen saturation, monitor heartbeat, and detect blood volume changes in the vascular bed. The advantages of PPG like compactness fast, and easy-to-operate [5-8] take the form of a portable instrument for routine screening. PPG signals can be obtained from multiple-site measurements including earlobe, forearm, fingertip, thumb, and toe [917]. A typical waveform of PPG is triphasic. Biphasic or monophasic form indicates a degree of arterial obstruction.

In signal processing, time-domain and frequency-domain analysis has been applied to assist diagnosis of PAD. Previous research reports that frequency parameters verify the differences between healthy and PAD subjects, using high-frequency and low-frequency characteristics [5-7,15]. The most common parameters of PPG waveforms are transit time, amplitude, and shape changes, which are helpful for evaluation of arterial compliance single or bilateral measurements of lower limbs. Previous research reports that these timing parameters and shape characteristics reflect the degree of vascular stenosis, contributing to time delay, damping, amplitude reduction, and changes of resistance and compliance properties of occlusive peripheral arteries, with variations depending on a subject's age and measurement sites [11, 13,15]. Therefore, an alternative for applying PPG to evaluate PAD is proposed, including bilateral or multichannel PPG techniques resulting from the findings of significant bilateral asymmetry and increased difference in timing and frequency parameters along with increased PAD severity [13-15,18]. Multi-channel measurements were used to study the age-related changes in the characteristics of PPG shape at the ear, finger, and toe sites. The largest changes with age were seen at the ear and finger sites for the systolic rising edge region, and the finger site for the dicrotic notch region. The smallest changes with age are for the toe site [9-11]. Accordingly, in this experiment, bilateral measurement at the right and left great toes was designed for PAD estimation.

The objective of this study was to construct a frequency-based Van der Pol (VDP) oscillator to evaluate the degree of PAD in patients with diabetes mellitus, and apply Burg autoregressive (AR) method as a parametric method for time-domain and frequency-domain signal processing [19-24], purporting to smooth the spectra and find characteristic frequencies from the PPG signals recorded. Given a bilateral frequency spectrum, a second- order VDP system has a step response that is characterized by damped oscillation [25-28]. The oscillation overshoot (OS) is an approximating function of the damped factor, and determines the severity of PAD: normal condition (Nor), mild-to-moderate disease (MD), and severe disease (SD). With twenty-one subjects, the test results demonstrate that the proposed method is efficient and accurate in the estimation of PAD in the feet of diabetics.

\section{FREQUENCY SPECTRA ANALYSIS}

In signal processing of plethysmographic waveforms, higher frequency characteristics are preferred for frequency analysis to identify normal pulse waves [5-7]. On the other hand, low frequency is used to characterize PAD in diabetic patients. Both frequency-based methods, such as Fourier transforms (FTs), and frequency spectrum methods, are the non-parametric techniques that are used to preprocess the PPG signals. They are disadvantageous for their spectral leakage effect, because of the size of sampling window. Wavelet transform is a parametric method and provides better time-frequency resolution than non-parametric methods, and would be a promising method to extract features from non-stationary biosignals. However, significant frequencies are extracted at specific wavelet coefficients with different wavelets, and several frequency bands are analyzed using the cascaded low-pass or high-pass filters and down-sampling/ up-sampling operations through trial procedures.

In literatures [17-20], the Burg autoregressive (AR) method is able to derive the frequency spectra by fitting an AR model of a given specific order. Its advantages over above-mentioned methods include smooth spectra, high frequency resolution, stable AR model, and computationally efficient. The Burg AR method is also a parametric method for time-domain and frequency-domain signal processes. It could smooth spectra in comparison with frequency-based methods to find the characteristic frequencies with distinguishing peak central/main spectra by fitting an AR model of a given specific order.

The frequency spectra of PPG signals is expressed as a linear combination of the previous samples and the residual values, res $_{i}$. For a discrete set of $n$ sampling points, $P$ coefficients are used to approximate the original data, $\phi_{i}, \quad i=1,2,3, \cdots, n$, where $\phi$ is a discrete frequency spectrum of the PPG signal. The residual value is assumed to be independent of the previous samples and is calculated by $[20,21]$

$$
\operatorname{res}_{i}=\phi_{i}+\sum_{p=1}^{P} \varepsilon_{p} \phi_{i-p}, \quad i=1,2,3, \cdots, n
$$

where $\phi_{i}$ is the frequency spectrum of the PPG signal, $P$ is the order of the AR model, and $p=1,2,3, \cdots, P$, and $\varepsilon_{p}$ are the coefficients of the AR model. 
The optimal coefficients, $\varepsilon_{p}$, are used to minimize the square error, $E=\sum_{n}\left(\text { res }_{i}\right)^{2}$, between the original data and the approximated data. For the forward and backward linear prediction, the object is to minimize $F_{p}$ and $B_{p}$, as shown in [22]

$$
\begin{aligned}
& F_{p}=\sum_{i=p}^{n}\left(\phi_{i}-\varphi_{t}\right)^{2}=\sum_{i=p}^{n}\left(\varepsilon_{0} \phi_{i}+\left(\sum_{t=1}^{p} \varepsilon_{i} \phi_{i-t}\right)\right)^{2}=\sum_{i=p}^{n}\left(f_{p}(i)\right)^{2} \\
& \text { with } f_{p}(i)=\sum_{i=0}^{p} \varepsilon_{i} \phi_{i-t} \\
& B_{p}=\sum_{i=p}^{n}\left(\phi_{i}-\lambda_{t}\right)^{2}=\sum_{i=0}^{n-p}\left(\varepsilon_{0} \phi_{i}+\left(\sum_{t=1}^{i} \varepsilon_{i} \phi_{i+t}\right)\right)^{2}=\sum_{i=0}^{n-p}\left(b_{p}(i)\right)^{2} \\
& \text { with } \quad b_{p}(i)=\sum_{i=0}^{p} \varepsilon_{i} \phi_{i+t}
\end{aligned}
$$

where $\varphi_{t}, t \in[p, n]$, is a linear weighted combination of $p$ previous sampling data, and $\lambda_{t}, t \in\left[0, n_{t-p}\right]$, is a linear weighted combination of $p$ subsequent sampling data. The sum of the residual energy (SORE) in stage $p$ is $E_{p}=$ $F_{p}+B_{p}$. The Levinson-Durbin recursion algorithm is used to minimize the SOREs to estimate the model coefficients. The coefficients of $\varepsilon_{p}, p \in[1, P]$, are stored in a vector $A_{p}=\left[1, \varepsilon_{1}, \varepsilon_{2}, \cdots, \varepsilon_{p}, \cdots, \varepsilon_{P}\right]^{\mathrm{T}}$ and an inverted order vector $V_{p}=\left[0, \varepsilon_{P}, \cdots, \varepsilon_{p}, \cdots, \varepsilon_{2}, \varepsilon_{1}, 1\right]^{\mathrm{T}}$. Therefore, the recursion formula is the following [22]

$$
\begin{gathered}
A_{p+1}=A_{p}+\mu V_{p} \\
\frac{\partial\left(F_{p+1}+B_{p+1}\right)}{\partial \mu}=0 \Rightarrow \mu=\frac{\sum_{i=0}^{n-p-1} f_{p}(i+p+1) b_{p}(i)}{\sum_{i=p+1}^{n} f_{p}(i)^{2}+\sum_{i=0}^{n-p-1} b_{p}(i)^{2}}
\end{gathered}
$$

The concept of Burg AR method adjusts the parameter $\mu$ to minimize the SORE $E_{p}$ using the final prediction error criterion (PEC) $[23,24]$. Then update the $A_{p}$ and update $f_{p}(i)$ and $b_{i}(i)$ as

$$
\begin{aligned}
& f_{p+1}(i)=f_{p}(i)+\mu b_{p}(i-p-1) \\
& b_{p+1}(i)=b_{p}(i)+\mu f_{p}(i+p+1)
\end{aligned}
$$

The optimal coefficients $\varepsilon_{p}$ are chosen to minimize the squared error, whereas the forward and backward linear prediction equations attempt to minimize $F_{p}$ and $B_{p}$. The entries of $\phi_{i}$ represent samples of a discrete data, and $P$ is the integer order of an AR model, used in estimating the power spectral density (PSD), $\log \left(\varphi_{i}\right) / \max \left\{\left|\log \left(\varphi_{i}\right)\right|\right\}$ $\mathrm{dB} / \mathrm{kHz}, \quad i=1,2,3, \cdots, n$. This property of the AR model is used to smooth the frequency spectrum, which tends to favor the peaky spectra. Qualitatively different PSDs are observed by normalized process, which different values within the specific ranges of normalized log-magnitude and scaled frequency. Therefore, PSD can be used to identify the characteristic frequencies and magnitudes in each frequency spectra.

In the physiological measurements, PPG signals were obtained using optical sensors $(940 \mathrm{~nm}$ near infrared, spectral bandwidth: $45 \mathrm{~nm}$ ) with $1 \mathrm{KHz}$ sampling rate, as shown in Figure 1(a). The dashed line represents a normal subject and the solid line represents a diabetic patient $[14,27,28]$. It was found that the Fourier transform based estimation methods has some drawbacks, such as spectral leakage into sidelobes and characteristic spectral broadening, as shown in Figures 1(b) and (c). In order to find characteristic frequencies, the Burg AR method is used to estimate the PSD by fitting an AR model with given specific orders, $P=8,12$, and 16 , to the PPG signals. The characteristic frequencies of the PPG signals easily found in the frequency spectra between $0 \mathrm{~Hz}$ and $500 \mathrm{~Hz}$. The PSDs are shown in Figures 1(d)-(f). The peaky spectra for diabetic subjects and the smooth frequency spectra for normal subjects fall into different frequency bands, respectively. Depending on these bands, frequency-based parameters provide information for the estimation of PAD in diabetic patients.

\section{THE ESTIMATION OF PERIPHERAL ARTERIAL DISEASE (PAD) SEVERITY}

\subsection{Feature Extraction Using the Van der Pol (VDP) Oscillator}

PAD can develop in the arteries of most visceral organs and extremities. Atherosclerosis in the brain and heart draws much attention for clinical diagnosis and intervention; by contrast, lower limb PAD is underestimated by the patients, even in diabetics whose progress of vascular atherosclerosis is accelerated by the metabolic disease. PPG is a noninvasive technique capable to optically generate a plethysmograph at great toes, index fingers, and ears, and monitor blood pressure, blood oxygen saturation $\left(\mathrm{SaO}_{2}\right)$, and blood volume changes in an artery or a vascular bed of tissue. A PPG signal consists of AC (Alternating Current) and DC (Direct Current) components. The AC component reveals physiological information, such as cardiac synchronous changes in the blood volume with each heartbeat and vasomotor activity [8-10, 15]. Time-domain and frequency-domain parameters have been used to detect the degree of PAD severity for diabetic patients with normal condition (Nor), mild-tomoderate disease (MD), and severe disease (SD). For the time-domain parameters, ECG R-peak as a timing reference is used to obtain pulse rise time (RT), pulse transit time (PTT) from R-peak to pulse foot $\left(\mathrm{PTT}_{\mathrm{f}}\right)$, pulse transit time from R-peak to pulse peak $\left(\mathrm{PTT}_{\mathrm{p}}\right)$, and PPG am- 

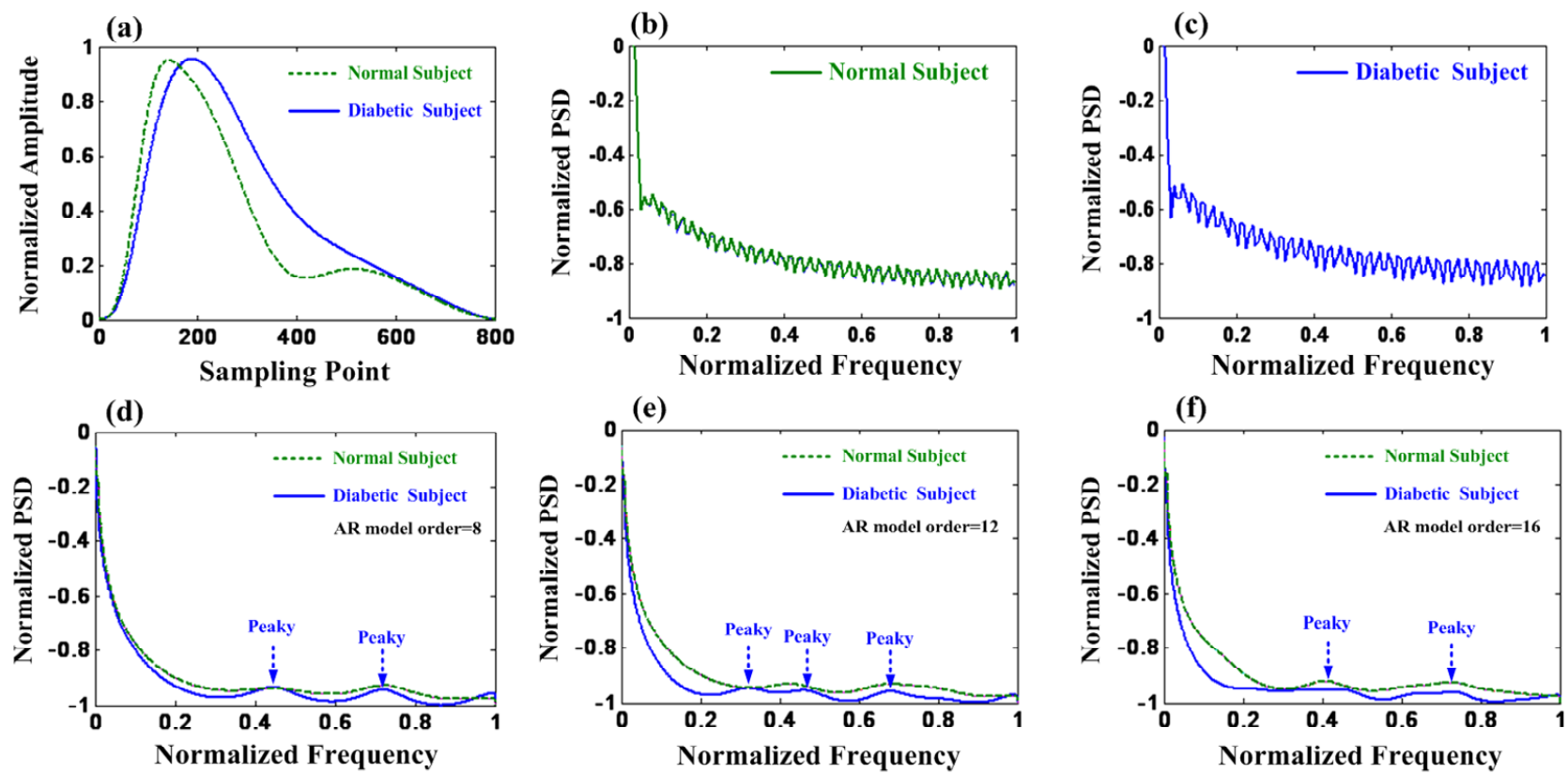

Figure 1. (a) Time-domain PPG signal analysis for normal subjects and diabetic subjects, (b) and (c) PSD estimation using the frequency spectrum method, (d), (e), and (f) PSD estimation using the Burg method for an AR model with orders $P=8,12$, and 16 for normal subjects and diabetic subjects.

plitude (plus foot-to-plus peak amplitude), as shown in Figure 2(a). The timing parameters, PTT and RT, are prolonged as due to the resistance of peripheral vessels increases, and the amplitude (AMP) and shape of pulse waves are smoothed [11]. Parameters, PTT and AMP, and shape differences vary with ages for individual sites, but the smallest variations with age occur at the great toe sites [9-15]. Thus, bilateral differences in the timing parameters, $\Delta \mathrm{PTT}_{\mathrm{f}}, \Delta \mathrm{PTT}_{\mathrm{p}}$, and $\Delta \mathrm{RT}$, provide time delay information for the estimation of PAD. Bilateral differences in frequency spectra are similar and are used to distinguish between the normal subjects and diabetic subjects.

This study uses frequency-based parameters to estimate the degree of PAD. The PSDs are estimated using the Burg AR method. The Van der Pol (VDP) system is used to quantify the features of the right- and left-side PSDs. It is an oscillator with nonlinear damping that is defined by a second-order differential equation. The formulation has the form of an autonomous system with two state variables. The state equations are evaluated as below [23-26]:

$$
\begin{gathered}
\frac{\mathrm{d} \phi_{r}(\omega)}{\mathrm{d} \omega}=\phi_{l}(\omega) \\
\frac{\mathrm{d} \phi_{l}(\omega)}{\mathrm{d} \omega}=-\phi_{r}(\omega)-\alpha\left[\left(\phi_{r}(\omega)\right)^{2}-1\right] \phi_{l}(\omega)
\end{gathered}
$$

where $\phi_{r}(\omega)$ and $\phi_{l}(\omega)$ are the functions with respect to frequency $\omega, \phi_{r}(\omega)$ is the PSD of the right-side PPG signal, except for $\omega=0$, and $\phi_{l}(\omega)$ is the PSD of the left- side PPG signal, right and left are denoted by the subscripts $r$ and $l$. The parameter, $\alpha$, is a control parameter, and $\alpha>0$ reflects the degree of nonlinearity of the system [21]. When the term, $\left(\phi_{r}(\omega)\right)^{2}$, becomes dominant, the VDP system becomes a nonlinear equation with positive damping. The dynamics of the system are stable and are restricted to a fixed point.

Equation (9) gives the frequency of self-oscillation, as determined by a real parameter, $\alpha$, and demonstrates dissipation or damping. If the parameter, $\alpha=0$, then Equation (9) reduces to that for a simple harmonic oscillator. When the PSDs, $\phi_{r}(\omega)$ and $\phi_{l}(\omega)$, are different (not symmetrical $), \phi_{l}(\omega)$ provides the damping in the VDP oscillator that results in self-sustained oscillations. The multiple peaks and amplitudes of these peaks demonstrate the nonlinearity of the frequency spectrum. PSD $\phi_{1}(\omega)$ is used to determine the response of the VDP oscillator. The VDP system demonstrates a damped sinusoidal response for a general second-order system. The transient response consists of a sinusoidal oscillating waveform with an exponentially decaying amplitude. The sinusoidal frequency is called the damped frequency of oscillation.

In order to evaluate the discrete frequency spectrum, the PSD is estimated using the Burg AR method. Assuming a set of $n$ points from the PSD, $\phi_{i}, i=1,2,3, \cdots, n$, ( $n=500$ in this study), the continuous VDP system can be modified as a discrete VDP system. Therefore, the discrete VDP system that is proposed for the estimation of PAD, as 


$$
\begin{gathered}
\Phi_{r}[i]=\phi_{l}[i] \\
\Phi_{l}[i]=-\phi_{r}[i]-\alpha\left[\left(\phi_{r}[i]\right)^{2}-1\right] \phi_{l}[i]
\end{gathered}
$$

where the index, $i$, is the integer scale of the frequency, $i=1,2,3, \cdots, n$. A bifurcation is a fundamental change in the nature of a solution. For different initial conditions, $\phi_{r}[i=1]$ and $\phi_{l}[i=1]$, the output, $\Phi_{l}[i]$, given by Equation (11), has a step response that is characterized by a damped oscillation in the frequency domain. It exhibits a rich variety of nonlinear dynamic behaviors and generates the limit cycle for small values of $\alpha$, and develops into relaxation oscillations when $\alpha$ becomes large. If the VDP oscillators use different PSDs, the oscillations become unstable, as the amplitudes of spectra peaks increase. The step response of the bilateral PSDs is shown in Figure 2. A second-order step response is characterized by damped oscillations. From Figure 2(c), the percentage overshoot $O S \%$ is given by the following Equation [29]:

$$
O S \%=\left(\frac{c_{\max }-c_{\min }}{c_{\min }}\right) \times 100 \%
$$

The percentage overshoot OS\% defines the amount by which the oscillation overshoots the minimum value $C_{\min }$ $=b$. The term $c_{\max }$ is determined by curve fitting the function at the maximum value, as [29]

$$
\left\{\begin{aligned}
c_{\max } & \approx b-\exp \left(-\left(\xi \pi / \sqrt{1-\xi^{2}}\right)\right)\left(\cos \pi+\frac{\xi}{\sqrt{1-\xi^{2}}} \sin \pi\right) \\
& =b+\exp \left(-\left(\xi \pi / \sqrt{1-\xi^{2}}\right)\right) \\
C_{\min } & =b
\end{aligned}\right.
$$

For the step response, substituting Equations (13) and (14) into Equation (12) gives

$$
O S \%=\frac{1}{b} \exp \left(-\left(\xi \pi / \sqrt{1-\xi^{2}}\right)\right) \times 100 \%
$$

Equation (15) demonstrates that the percentage overshoot is a function only of the index, $\xi$, and allows the determination of the $O S \%$, given the index, $\xi$. The inverse of the equation allows a solution for $\xi$ given the $O S \%$. The inverse is given by

$$
\xi=\frac{-\ln (b \times O S \%)}{\sqrt{\pi^{2}+\ln ^{2}(b \times O S \%)}}
$$

The relationship between the index, $\xi$, and the multiple characteristic frequencies is clearly seen. The higher the value of $\xi$, the more oscillatory is the response in the frequency-domain from Equations (10), (11), (15), and

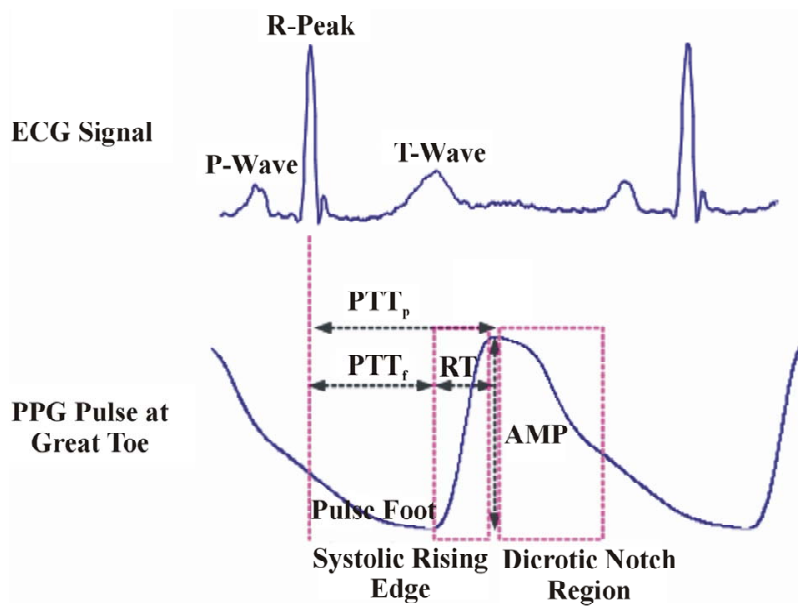

(a)

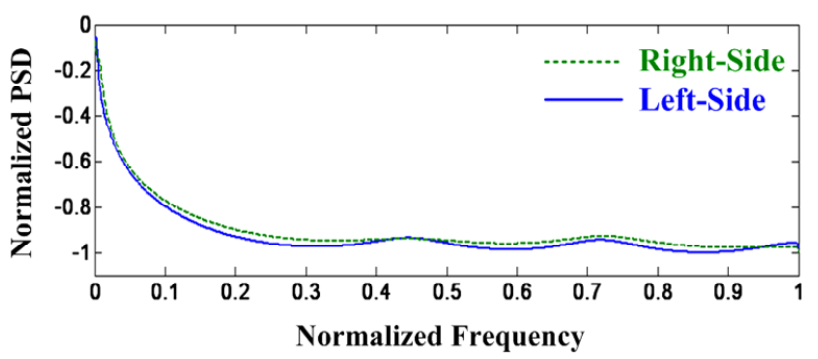

(b)

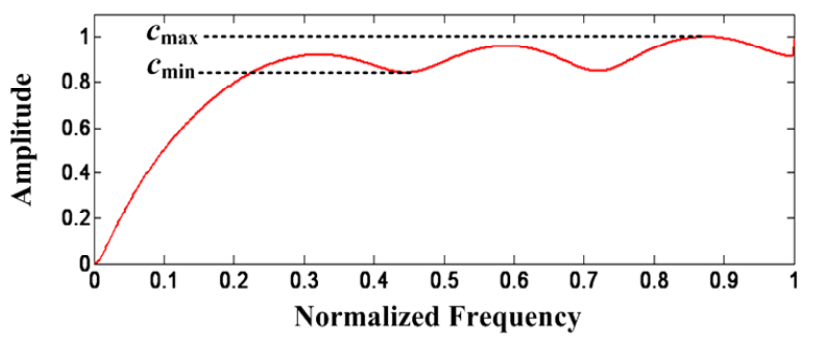

(c)

Figure 2. (a) ECG and PPG signals and pulse landmarks, (b) The bilateral PSD functions for right-side and left-side PPG signals, (c) The damped sinusoidal response.

(16). The increase in the resistance of peripheral vessels produces multiple characteristic frequencies, so this study uses the index, $\xi$, is used to estimate the degree of PAD. The various ranges of the index $\xi$ are obtained from specific subjects, including those with normal condition (Nor), mild-to-moderate disease (MD), and severe disease (SD).

\subsection{Physiological Measurement}

PPG signals were collected from twenty-one subjects in a hospital (Chi-Mei Medical Center, Department of Infectious Disease and Immunology, Tainan City, Taiwan). The subjects, aged from 24 to 65 years, were divided into three groups: Nor, MD and SD, in terms of diabetic disease. In a clinical examination, the ABPI (Ankle-Bra- 
chial Pressure Index) was used as an early detection method to decide the degree of PAD. The degrees were categorized by clinical manifestations, ABPI values, and angiographic findings. For preliminary PAD estimation using the APBI, the indices signify ABPI $\geq 0.9$ for normal subjects and diabetic subjects with MD, ABPI $<0.9$ (at least one leg) for diabetic subjects with SD, as shown in Table 1 [28-30,32]. Despite the non-invasive nature of the examination to assess death rate and cardiovascular diseases in high-risk patients, the accuracy of the ABPI measurement is reduced in patients with calcified blood vessels, caused by conditions such as diabetes and chronic renal failure. However, measurement of the ABPI must be repeated several times ( $>10$ minutes) and is of limited use in routine vascular screening in a primary care setting [13].

Bilateral timing parameters could offer a quick assessment for the screening of PAD in primary care. Bilateral measurements simultaneously acquire PPG signals from the right and left big toes. The absolute timing differences are used to reference one side of the body (right side) with the contra-lateral side (left side), in order to calculate the parameters, $\Delta \mathrm{PTT}_{\mathrm{f}}, \Delta \mathrm{PTT}_{\mathrm{p}}$ and $\Delta \mathrm{RT}$, so various ranges are obtained for specific groups. Each parameter has a mean value and a specific range between maximum (Max) and minimum (Min) values. A comparison of the bilateral differences demonstrates that these parameters increase as the severity of the disease increases. Therefore, clinical physicians would consider timing differences to be a good reference for PAD assessment. The three groups are divided into ten normal subjects (No. 1 - No. 10), eight MD diabetic subjects (No. 11 - No. 18) and three SD diabetic subjects (No. 19 - No. 21), as shown in Table 1.

For these twenty-one subjects, Equations (12) and (16) were used to compute the index, $\xi$. The indices, $\xi$, also represent the specific ranges for diabetic patients, nondiabetic patients with PAD and normal subjects as a control, as shown in Figure 3. Severe PAD causes more asymmetrical PPG signals and the damped oscillation increases as PAD increases, which is caused by the resistance of the peripheral vessels. Therefore, the index, $\xi$, also increases in diabetic patients with increased PAD. The index, $\xi$, is less than 0.64 for normal subjects with an ABPI $\geq 0.9$, from 0.64 to 0.65 in diabetic patients with an ABPI $\geq 0.9$ and greater than 0.65 for diabetic patients with an ABPI $<0.9$. The standard indices, $\xi$, are established, as shown in Table 1. The sensitivity is greater than $85.7 \%$, and positive predictivity is also greater than $80.0 \%$ to quantify the performance of the proposed measurement method. These specific ranges also confirm the degree of PAD noted by clinical physicians. The trend in the degeneration of PAD is clearly demonstrated. Depending on the severity of the PAD assessment, these specific ranges provide key information for the evaluation of the degree of PAD.

\section{EXPERIMENTAL RESULTS AND DISCUSSION}

\subsection{Experimental Setup}

Using the bilateral non-invasive measurement, two optical sensors (reflection mode), consisting of light sources, photo-detectors, trans-impedance amplifiers, and highpass filters, were placed at the right and left great toes. Near infrared (NIR) has large differences in the extinction coefficients of deoxyhaemoglobin and oxyhaemoglobin. Thus, the light source $940 \mathrm{~nm}$ NIR was chosen in this optoelectronic design. The reflection mode, including light source and photo-detector, was positioned side by side with $5 \mathrm{~mm}$ spacing, and the light is directed down into the skin and is backscattered from the skin adjacent to the photo-detector [10]. Two optoelectronic probes (circle shape, diameter: $20.0 \mathrm{~mm}$, height: $8.2 \mathrm{~mm}$ ) were synchronized using a data acquisition controller. PPG signals are captured at a sampling rate of $1 \mathrm{kHz}$ for 15 minutes [30]. A DAQ card (National Instruments DAQ Card, 16 Channels, $1.25 \mathrm{MS} / \mathrm{s}$ ) served as an analog-to-digital (A/D) converter between the optical measurement system and a computer. Locating each pulse foot (PF)-pulse foot (PF) interval of PPG signal, 800 sampling data were acquired within a sampling window. In this study, we used the Burg AR method to estimate the PSDs of PPG signals. The suitable AR order can be used to identify the peaky spectra. Figure 4 shows the variation of residual energy versus AR model order. We use model orders, from order 1 to order 30 , to calculate the SORE. To obtain the suitable order, Akaike's final prediction criterion is used to select the model order $[22,24]$. For considering convergent condition, we considered SORE $\leq 10^{-1}$ to stop the Burg AR algorithm. Then, the Burg method using model order $P=8$ with optimal coefficients was used to estimate the PSDs.

The VDP oscillator shows the step responses in the frequency-domain, as shown in Figure 5. The oscillations of the step responses are smaller than those for normal subjects. The higher the value of $\xi$, the more oscillatory the response are important information for the degree of PAD assessment, which may be useful for determining the trends of PAD in routine examination. The proposed algorithms were developed on a PC AMD Celeron (R) CPU 2.40GHz with $2.39 \mathrm{GHz}, 224 \mathrm{MB}$ RAM GHz with 1.75 GB RAM and Matlab software. Portable optical measurement was used to obtain the PPG signals in the laboratory and medical center. To demonstrate the effectiveness of the proposed method, the database of 21 subjects were used to design the gold standard of PAD assessment using the VDP oscillator from selected 6 subjects 
Table 1. The ABPI, bilateral differences in timing parameters [30], and index $\xi$ for PAD estimation.

\begin{tabular}{|c|c|c|c|c|}
\hline \multirow{3}{*}{ Parameter } & \multirow{3}{*}{$\begin{array}{l}\text { Subject } \\
\text { Category }\end{array}$} & $\begin{array}{l}\mathrm{ABPI} \geq 0.9 \\
\quad(10)\end{array}$ & $\begin{array}{c}0.5 \leq \mathrm{ABPI}<0.9 \\
(8)\end{array}$ & $\begin{array}{l}\mathrm{ABPI}<0.5 \\
\quad(3)\end{array}$ \\
\hline & & Normal subject & Diabetic subject & Diabetic subject \\
\hline & & Normal (Nor) & Mild-to-moderate disease (MD) & Severe disease (SD) \\
\hline$\Delta \mathrm{PTT}_{\mathrm{f}}(\mathrm{ms})$ & $\begin{array}{c}\text { Mean } \\
\text { Min-Max }\end{array}$ & $\begin{array}{c}2.58 \\
0.3-7.4\end{array}$ & $\begin{array}{c}9.2 \\
5.1-23.7\end{array}$ & $\begin{array}{c}29.2 \\
23.6-34.8\end{array}$ \\
\hline$\Delta \mathrm{PTT}_{\mathrm{P}}(\mathrm{ms})$ & $\begin{array}{c}\text { Mean } \\
\text { Min-Max }\end{array}$ & $\begin{array}{c}7.3 \\
0.4-22.3\end{array}$ & $\begin{array}{c}22.6 \\
14.3-56.5\end{array}$ & $\begin{array}{c}52 \\
46.2-57.8\end{array}$ \\
\hline$\Delta \mathrm{RT}(\mathrm{ms})$ & $\begin{array}{c}\text { Mean } \\
\text { Min-Max }\end{array}$ & $\begin{array}{c}6.84 \\
1.3-15.6\end{array}$ & $\begin{array}{c}14.6 \\
3.4-32.3\end{array}$ & $\begin{array}{c}23.4 \\
11.5-35.3\end{array}$ \\
\hline Index $\xi$ & $\begin{array}{c}\text { Mean } \\
\text { Min-Max }\end{array}$ & $\begin{array}{c}0.616 \pm 0.014 \\
0.597-0.636\end{array}$ & $\begin{array}{l}0.645 \pm 0.002 \\
0.640-0.647\end{array}$ & $\begin{array}{l}0.668 \pm 0.012 \\
0.652-0.681\end{array}$ \\
\hline
\end{tabular}

Note: The values of $\Delta \mathrm{PTT}_{\mathrm{f}}, \Delta \mathrm{PTT}_{\mathrm{p}}$, and $\Delta \mathrm{RT}$ are absolute values. (1) $\Delta \mathrm{PTT}_{\mathrm{f}}=\left|\mathrm{PTT}_{\mathrm{Rf}}-\mathrm{PTT}_{\mathrm{Lf}}\right|$, (2) $\Delta \mathrm{PTT}_{\mathrm{p}}=\left|\mathrm{PTT}_{\mathrm{Rp}}-\mathrm{PTT}_{\mathrm{Lp}}\right|$, (3) $\Delta \mathrm{RT}=\left|\mathrm{RT}_{\mathrm{R}}-\mathrm{RT}_{\mathrm{L}}\right|$, where suffix words $\mathrm{R}$ and $\mathrm{L}$ are defined right and left legs, (4) ABPI: it is calculated using the highest of the right and left ankle systolic blood pressure divided by the highest of the right and left arm brachial systolic blood pressure [13].

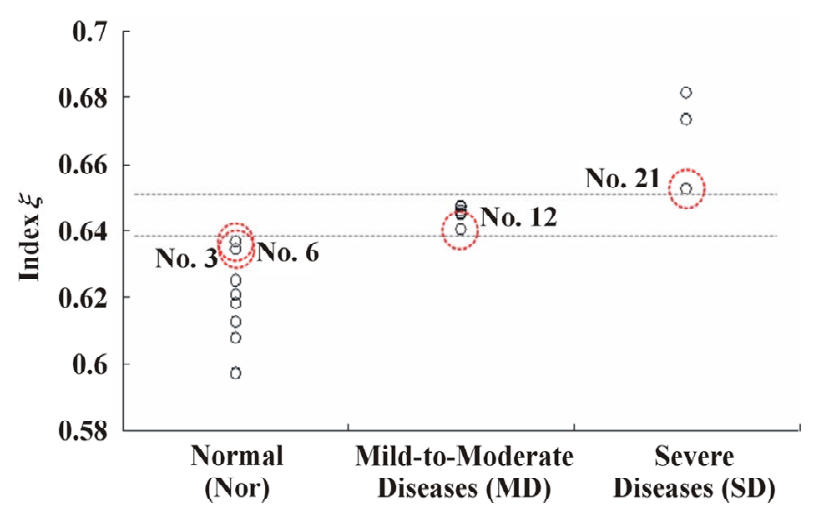

Figure 3. The specific ranges of the index $\xi$ in normal subjects and diabetic patients with PAD.

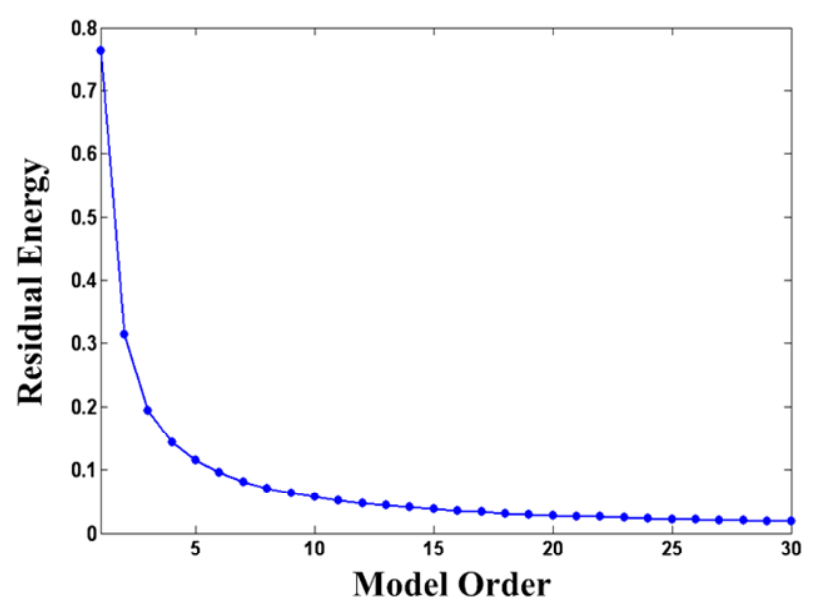

Figure 4. Variation of residual energy versus AR model order.

(three groups: Nor, MD, and SD) were used to test, as shown in Table 2. The result demonstrates the computational efficiency and accurate diagnosis achieved by this study.

\subsection{Experimental Results}

In order to verify the proposed method, normal subjects
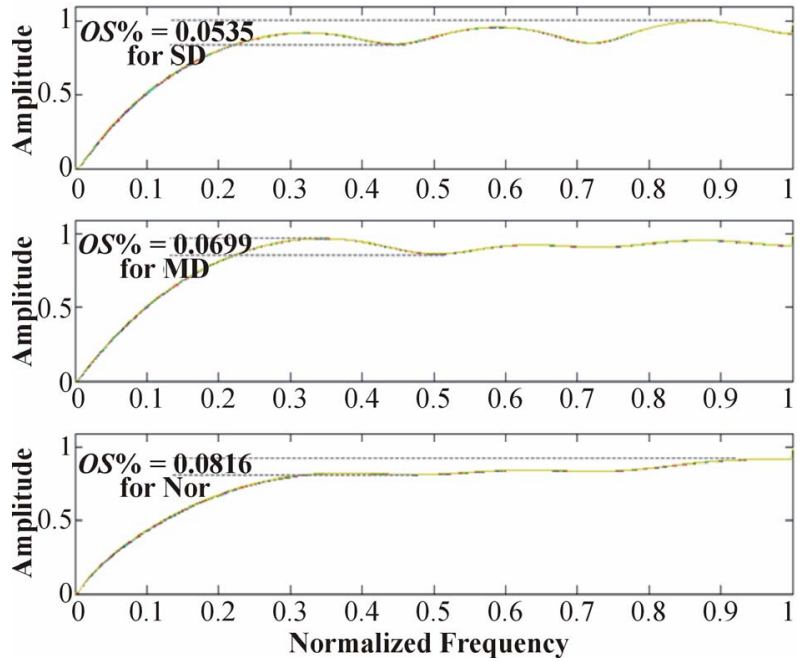

Figure 5. The step responses of the VDP Oscillator for severe disease (SD), mild-to-moderate disease (MD), and normal condition (Nor).

and diabetic patients with PAD were tested using physiological measurement. There were two normal subjects (No. 1 and No. 2), four diabetic patients, two of which were MD subjects (No. 11 and No. 12) and two of which were SD subjects (No. 19 and No. 20). The preliminary diagnosis and ABPI classification were performed by clinical physicians and the results are shown in Table 2. However, the ABPI is significantly lower for normal subjects and diabetic patients with $\mathrm{MD}(\mathrm{ABPI} \geq 0.9)$. For bilateral PPG measurement, two optical sensors were placed on the right and left great toes and PPG signals were obtained from the three groups, as shown in Figure 6. The transit time is prolonged for an occluded leg and gradually increases as the disease becomes more severe, as seen in the interval between the dashed lines. For example, the bilateral-timing differences are

Normal Subject-No. 1: $\Delta \mathrm{PTT}_{\mathrm{f}}=2.733, \Delta \mathrm{PTT}_{\mathrm{p}}=$ $1.977, \Delta \mathrm{RT}=3.133$, 
Table 2. The experimental results of PAD estimation.

\begin{tabular}{|c|c|c|c|c|c|c|c|c|c|c|}
\hline \multirow{2}{*}{$\begin{array}{c}\text { Subject } \\
\text { No. }\end{array}$} & \multicolumn{3}{|c|}{ Mean values of bilateral differences } & \multirow{2}{*}{$\begin{array}{l}\text { ABPI } \\
\text { R-Leg }\end{array}$} & \multirow{2}{*}{$\begin{array}{l}\text { ABPI } \\
\text { L-Leg }\end{array}$} & \multirow{2}{*}{$\begin{array}{l}\text { Clinical } \\
\text { physician } \\
\text { decision }\end{array}$} & \multirow{2}{*}{$\begin{array}{l}\text { Bilateral } \\
\text { timing } \\
\text { difference }\end{array}$} & \multirow{2}{*}{$\begin{array}{l}\text { ANFIS } \\
\text { decision } \\
\text { (output) }\end{array}$} & \multirow{2}{*}{$\begin{array}{c}\text { Fuzzy logic } \\
\text { decision } \\
\text { (output) }\end{array}$} & \multirow{2}{*}{$\begin{array}{l}\text { The proposed } \\
\text { method } \\
\text { (index } \xi \text { ) }\end{array}$} \\
\hline & $\Delta \mathrm{PTT}_{\mathrm{f}}(\mathrm{ms})$ & $\Delta \mathrm{PTT}_{\mathrm{p}}(\mathrm{ms})$ & $\Delta \mathrm{RT}(\mathrm{ms})$ & & & & & & & \\
\hline 1 & $2.7330 \% \pm 7.05 \%$ & $1.9970 \% \pm 16.26 \%$ & $3.1330 \% \pm 0.19 \%$ & 1.0650 & 1.1138 & Nor & Nor & $\begin{array}{c}\text { Nor } \\
(0.1988)\end{array}$ & $\begin{array}{c}\text { Nor } \\
(0.1750)\end{array}$ & $\begin{array}{c}\text { Nor } \\
(0.6199)\end{array}$ \\
\hline 2 & $1.1730 \% \pm 2.78 \%$ & $5.8700 \% \pm 4.05 \%$ & $4.6950 \% \pm 3.97 \%$ & 1.1404 & 1.1333 & Nor & Nor & $\begin{array}{c}\text { Nor } \\
(0.1990)\end{array}$ & $\begin{array}{c}\text { Nor } \\
(0.1620)\end{array}$ & $\begin{array}{c}\text { Nor } \\
(0.6223)\end{array}$ \\
\hline 3 & $2.3330 \% \pm 12.07 \%$ & $3.6660 \% \pm 5.44 \%$ & $13.330 \% \pm 3.97 \%$ & 1.1440 & 1.1101 & Nor & Nor & $\begin{array}{c}\text { Nor } \\
(0.1978)\end{array}$ & "Failure & $\begin{array}{c}\text { Nor } \\
(0.6336)\end{array}$ \\
\hline 11 & $15.321 \% \pm 16.92 \%$ & $17.286 \% \pm 10.50 \%$ & $1.9640 \% \pm 4.05 \%$ & 1.1788 & 1.2357 & MD & MD & $\begin{array}{c}\text { MD } \\
(0.4000)\end{array}$ & $\begin{array}{c}\text { MD } \\
(0.4000)\end{array}$ & $\begin{array}{c}\text { MD } \\
(0.6451)\end{array}$ \\
\hline 12 & $6.3850 \% \pm 13.44 \%$ & $16.923 \% \pm 16.76 \%$ & $17.556 \% \pm 0.39 \%$ & 1.2735 & 1.3076 & MD & MD & $\begin{array}{c}\text { MD } \\
(0.3988)\end{array}$ & $\begin{array}{c}\text { MD } \\
(0.3830)\end{array}$ & $\begin{array}{c}\text { MD } \\
(0.6478)\end{array}$ \\
\hline 13 & $23.667 \% \pm 13.62 \%$ & $33.333 \% \pm 7.58 \%$ & $32.833 \% \pm 16.63 \%$ & 1.0714 & 1.0446 & MD & *SD & $\begin{array}{c}\text { MD } \\
(0.4000)\end{array}$ & $\begin{array}{c}\text { MD } \\
(0.4020)\end{array}$ & $\begin{array}{c}\text { MD } \\
(0.6476)\end{array}$ \\
\hline 19 & $33.072 \% \pm 10.06 \%$ & $48.500 \% \pm 14.18 \%$ & $15.428 \% \pm 8.57 \%$ & 1.0817 & 0.8941 & SD & SD & $\begin{array}{c}\text { SD } \\
(0.6000)\end{array}$ & $\begin{array}{c}\text { SD } \\
(0.6000)\end{array}$ & $\begin{array}{c}\text { SD } \\
(0.6733)\end{array}$ \\
\hline 20 & $23.606 \% \pm 13.64 \%$ & $57.700 \% \pm 6.05 \%$ & $35.000 \% \pm 10.83 \%$ & 1.0442 & 0.8945 & SD & SD & $\begin{array}{c}\text { SD } \\
(0.5988)\end{array}$ & $\begin{array}{c}* \mathrm{MD} \\
(0.4000)\end{array}$ & $\begin{array}{c}\text { SD } \\
(0.6810)\end{array}$ \\
\hline
\end{tabular}

Note: ANFIS and Fuzzy Logic: 1 output variable with 3 triangular membership functions, the center values of the functions are 0.2, 0.4, and 0.6 for Nor, MD, and SD.

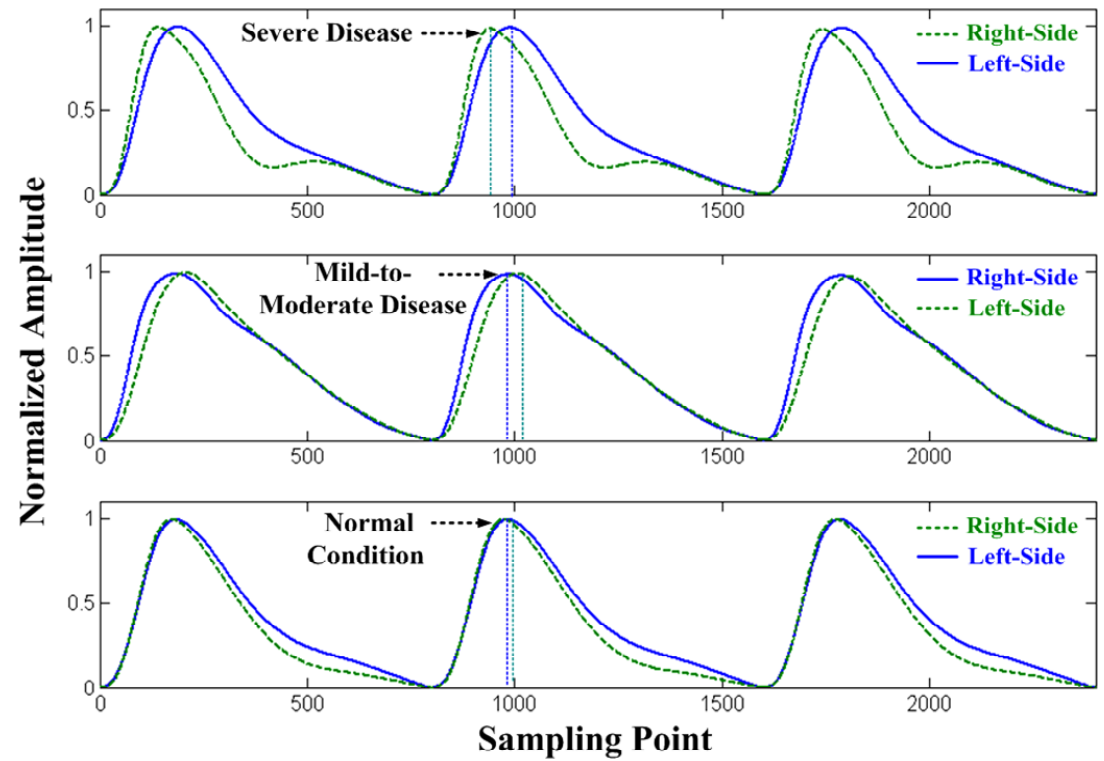

Figure 6. Time-domain PPG signals of the three groups of patients.

MD Subject-No. 11: $\Delta \mathrm{PTT}_{\mathrm{f}}=15.321, \Delta \mathrm{PTT}_{\mathrm{p}}=$ 17.286, $\Delta \mathrm{RT}=1.964$,

$\square \quad$ SD Subject-No. 19: $\Delta \mathrm{PTT}_{\mathrm{f}}=33.072, \Delta \mathrm{PTT}_{\mathrm{p}}=48.500$, $\Delta \mathrm{RT}=15.428$.

The shape of the PPG signals for a SD subject with a unilateral occluded leg is substantially different. The PPG signals at the right and left sites are asynchronous in the time-domain. According to the standard established in Table 1 [30], the degree of PAD from the preliminary estimate is confirmed, as shown in Table 2. However, the ABPI and bilateral timing differences are not the only parameters that determine classification.

Using twenty bilateral PPG signals, the diagnostic results for six subjects are shown in Figure 7, where the horizontal axis is the number of PPG signals and the vertical axis is the index, $\xi$, for each PAD assessment. The Burg AR method is used to estimate the frequency spectra from the bilateral PPG signals. Using the bilateral frequency spectra, the VDP oscillator shows the dynamic characteristics to be step damped oscillation responses for diabetic patients with PAD. In this study, the index, $\xi$, is a parameter that represents the degree of PAD that is 


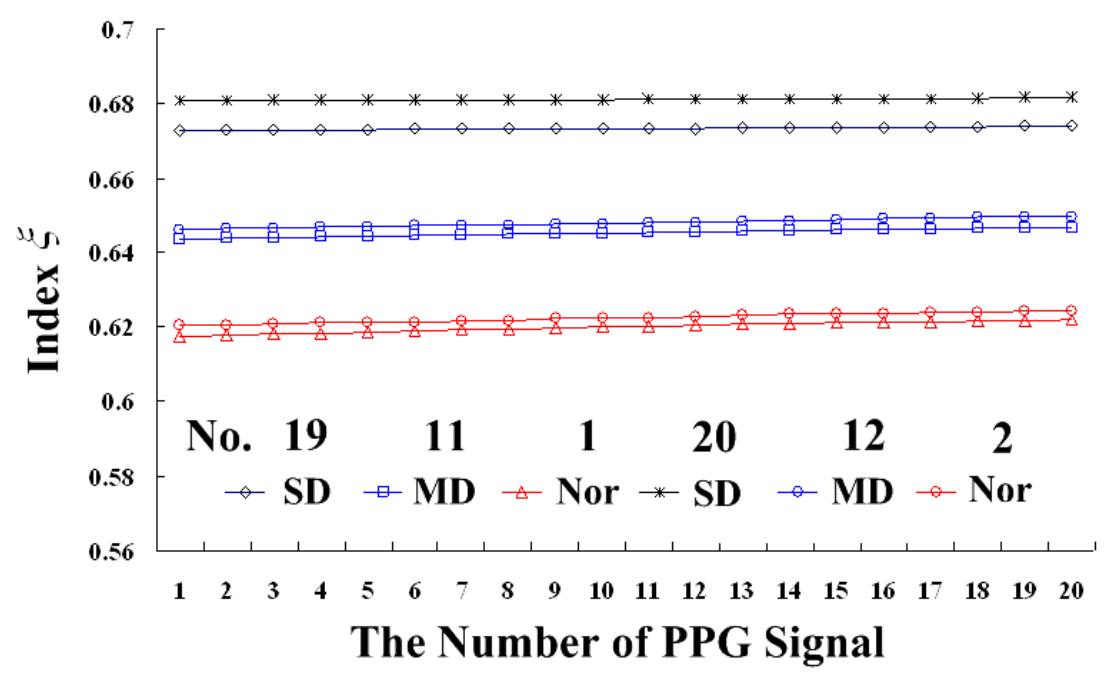

Figure 7. Different indexes $\xi$ for separating the diabetic PAD from the normal subjects.

assessed using the VDP oscillator, for the Nor, MD and SD groups. A comparison of the results shown in Figure 7, demonstrates that the index, $\xi$, is a significant parameter for the separation of diabetic patients with PAD from normal subjects. According to the standard values in Table 1, the values are centered at $0.645( \pm 0.002)$ for MD subjects and are greater than 0.65 for SD subjects $(0.668 \pm 0.012)$. Within these specific ranges, the index, $\xi$, can also be used to estimate the degree of PAD.

Experimental tests show that the proposed VDP oscillator can be used to assess the degree of severity and can be used to monitor the trends in PAD degeneration at the borders between Nor and MD (subjects No. 3, No. 6, and No. 12), or between MD and SD (subject No. 21). In this study, the index, $\xi$, is used to monitor the trends in the degeneration of PAD to allow early detection or screening and monitoring. Diabetes mellitus is a chronic disease that may require therapy to prevent further problems. In particular, for the feet of diabetics, adequate treatment may improve the risk profile of chronic complications. This research provides a potential method for early detection, monitoring, and prevention of PAD by early intervention to control its risk factors.

\subsection{Discussions}

Table 2 shows a comparison of the experimental results using the proposed method, bilateral timing difference, Fuzzy logic decision, and an adaptive network based fuzzy inference system (ANFIS). Using the bilateral timing difference, the timing parameters, $\Delta \mathrm{PTT}_{\mathrm{f}}, \Delta \mathrm{PTT}_{\mathrm{p}}$ and $\triangle \mathrm{RT}$, are used to estimate the degrees of PAD severity. For example, subjects, No. 2 and No. 12, were confirmed as a healthy subject and a MD diabetic patient, respectively. According to the Table 1, the timing parameters, $\Delta \mathrm{PTT}_{\mathrm{f}}=6.3850$ and $\Delta \mathrm{PTT}_{\mathrm{p}}=16.923$, signify an overlap between Nor (standard: $\Delta \mathrm{PTT}_{\mathrm{f}}=0.3-7.4$ and $\Delta \mathrm{PTT}_{\mathrm{p}}=0.4-22.3$ ) and MD (standard: $\Delta \mathrm{PTT}_{\mathrm{f}}=5.1-$ 23.7 and $\Delta \mathrm{PTT}_{\mathrm{p}}=14.3-56.5$ ). If there is overlapping of the ranges of the timing parameters, the inferences are affected by the timing reference (heart rate) and measurement errors. This demonstrates that timing parameters alone are insufficient for the estimation of the degree of PAD. The clinical physicians decided the possible degree using a combination of the variances in the three timing parameters. However, this examination method required off-line analysis to obtain a diagnosis.

According to the Table 1, each timing parameter has a mean value and a specific range between the minimum (Min) and maximum (Max) values. A triangular membership function is parameterized by its triplet values (Min, Mean, Max) and is decomposed into three fuzzy partitions, defined as Nor, MS and SD, as shown in Figure 8. Therefore, the Fuzzy logic decision has three input variables with nine triangular membership functions and 1 output variable with three triangular membership functions. The fourteen linguistic Fuzzy rules for the three degrees are determined by professional physicians over many examinations, as shown in Table 1. Inference uses the centre of the mean of the maximal defuzzifier. For adaptive and self-organizing applications, the literature $[30,31,33,34]$ also cites the gradient descent method, the least-square algorithm and the modified least-square algorithm for training a network structure and network parameters. The ANFIS structure is immediately determined after the presentation of each input-output pair and the inference rules. Updating of the parameters is then performed after the network structure has been decided. In this study, the least-square algorithm is used to tune network parameters. For the same subjects, the overall results are shown in Table 2. The sensitivity is $76.2 \%$ using Fuzzy logic decision. The ANFIS has more 

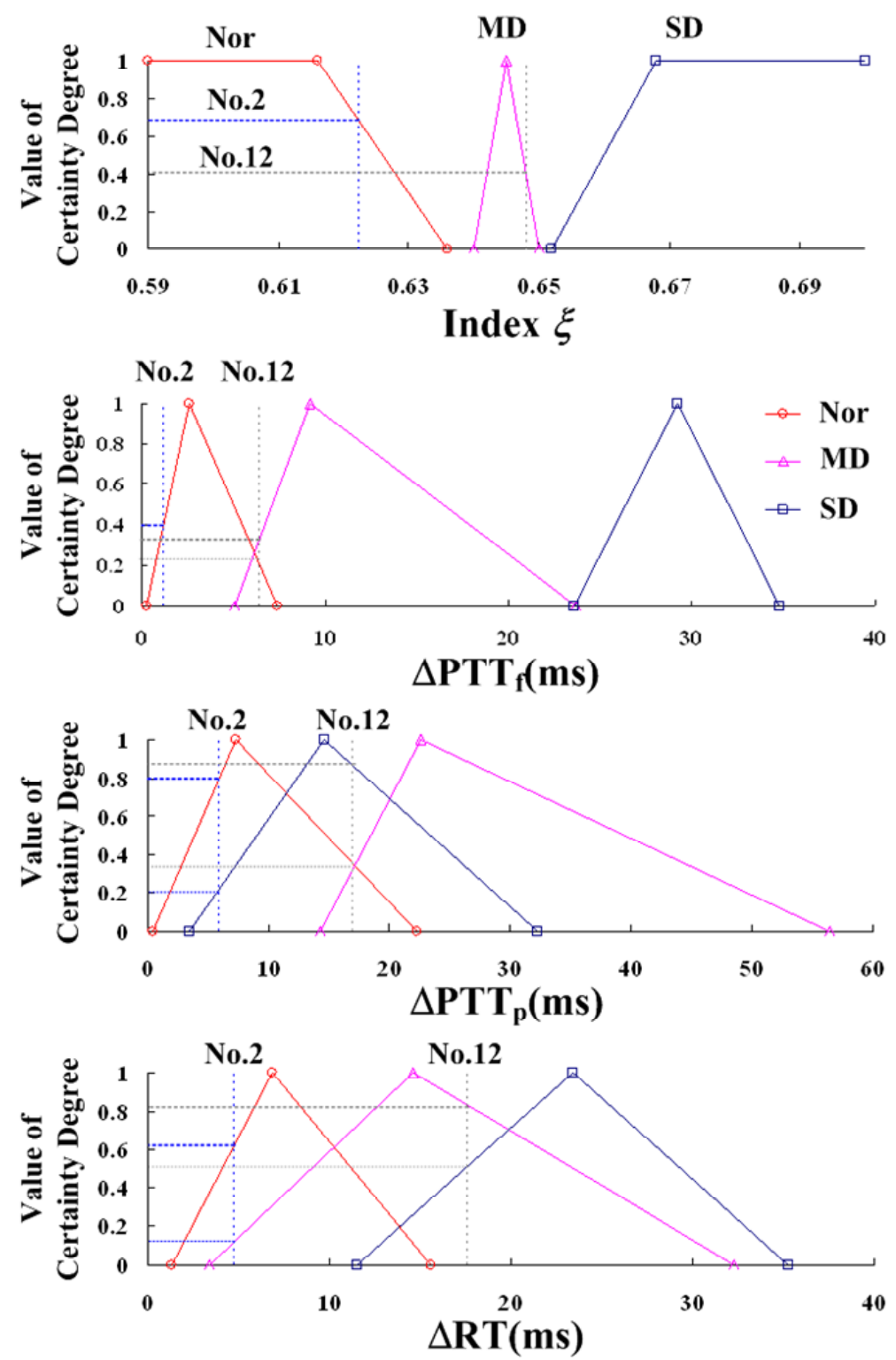

Figure 8. The value of certainty degree versus index, $\xi$, and bilateral timing parameters for separating the diabetic PAD.

than $90 \%$ of sensitivity, but the adaptive mechanism is difficult implement in hardware devices, because of needs to assign and update network parameters [35].

The experimental results validate the effectiveness of the proposed VDP oscillator. A comparison with the proposed method and other examination methods, the results of the 21 patients demonstrate the $85.7 \%$ of sensitivity and allow early detection between Nor and MD or between MD and SD. However, the trends of the index, $\xi$, increases as the PAD gradually becomes severity. The proposed screening method provides a finding to evaluate the orders of PAD for a routine examination. Its advantages are summarized as follows.

- The Burg AR method overcomes spectral leakage and smoothes the spectra to determine the characteristic frequencies.

- The proposed algorithm for the VDP oscillator is easily implemented in hardware devices, such as embedded system (ES) and field-programmable gate array (FPGA) chips.

- The proposed VDP oscillator can also be implemented using analog electronic circuits.

The proposed method potentially allows the use of a portable monitor for the estimation of diabetic foot PAD in daily homecare.

\section{CONCLUSION}

The Van der Pol (VDP) oscillator was able to detect pho- 
toplethysgraphic signals to estimate severity of peripheral arterial disease (PAD) in patients with diabetes mellitus. In addition, bilateral non-invasive optical sensors were helpful for acquiring the PPG signals at the right and left great toes, combined with the Burg AR method for determining the frequency spectra of the right- and left-side PPG signals. Using the bilateral frequency spectra, the VDP oscillator shows the step damped oscillation responses for diabetic patients with mild and severe severity, respectively, with the increasing amplitudes of damped oscillation as PAD severity worsening. The oscillation amplitude is expressed as an index $\xi$ to differentiate between diabetic patients with PAD and normal subjects. The numerical experiments reveal specific ranges that allow confirmation of the degree, which allows tracking the trends of PAD for screening and monitoring of high-risk patients in primary settings as well as in hospitals. For an improved diagnostic design, the proposed method can be combined with a fuzzy inference system to derive an index for the diagnosis of PAD. The proposed method potentially allows for constructing a portable bio-monitor and the use of telemedicine in a variety of clinical environments.

\section{ACKNOWLEDGEMENTS}

This work is supported in part by the National Science Council of Taiwan under contract number: NSC99-2221-E-244-007 (August 1 2010October 31 2011). The authors would like to thank Dr. Chian-Ming Li for providing his valuable suggestion and help on experiments.

\section{REFERENCES}

[1] I. S. Muller, M. L. Bartelink, W. J. C. Grauw, H. J. M. Hooden, W. H. E. M. Gerwen and G. E. H. M. Rutten, "Foot Ulceration and Low Limb Amputation in Type 2 Diabetic Patients in Dutch Primary Health Care," Diabetes Care, Vol. 25, No. 3, 2002, pp. 570-574. http://dx.doi.org/10.2337/diacare.25.3.570

[2] J.-Y. David, S. A. Jones and D. P. Giddens, "Modern Spectral Analysis Techniques for Blood Flow Velocity and Spectral Measurements with Pulsed Doppler Ultrasound," IEEE Transactions on Biomedical Engineering, Vol. 38, No. 6, 1991, pp.589-596. http://dx.doi.org/10.1109/10.81584

[3] C. Loewe, M. Schoder, T. Rand, U. Hoffmann, J. Sailer, T. Kos, J. Lammer and S. Thurnher, "Peripheral Vascular Occlusive Disease: Evaluation with Contrast-Enhanced Moving-Bed MR Angiography versus Digital Subtraction Angiography in 106 Patients," American Journal of Roentgenology, Vol. 179, No. 4, 2002, pp. 1013-1021. http://dx.doi.org/10.2214/ajr.179.4.1791013

[4] O. Pablo Vasquez, M. Marco Munguia and B. Mandersson, "Arteriovenous Fistula Stenosis Detection Using Wavelets and Support Vector Machines," 31st Annual International Conference of the IEEE EMBS, Mineapolis, 2-6 September 2009, pp. 1298-1301.
[5] M. Nitzan, A. Babchenko and B. Khonokh, "Very Low Frequency Variability in Arterial Blood Pressure and Blood Volume Pulse," Medical \& Biological Engineering \& Computing, Vol. 37, No. 1, 1999, pp. 54-58. http://dx.doi.org/10.1007/BF02513266

[6] E. D. Übeyli, D. Cvetkovic and I. Cosic, "AR Spectral Analysis Technique for Human PPG, ECG and EEG Signals," Journal of Medical Systems, Vol. 32, No. 3, 2008, pp. 201-206.

http://dx.doi.org/10.1007/s10916-007-9123-7

[7] Y. M. Akay, M. Akay, W. Welkowitz, S. Lewkowicz and J. L. Semmlow, "Non Invasive Acoustical Detection of Coronary Artery Disease: A Comparative Study of Signal Processing Methods," IEEE Transactions on Biomedical Engineering, Vol. 40, No. 6, 1993, pp. 571-578. http://dx.doi.org/10.1109/10.237677

[8] J. Allen, "Photoplethysmography and Its Application in Clinical Physiological Measurement," Physiological Measurement, Vol. 28, No. 3, 2007, pp. R1-R39. http://dx.doi.org/10.1088/0967-3334/28/3/R01

[9] J. Allen and A. Murray, "Age-Related Changes in the Characteristics of the Photoplethysmographic Pulse Shape at Various Body Sites," Physiological Measurement, Vol. 24, No. 2, 2003, pp. 297-307. http://dx.doi.org/10.1088/0967-3334/24/2/306

[10] R. Erts, J. Spigulis, I. Kukulis and M. Ozols, "Bilateral Photoplethysmography Studies of the Leg Arterial Stenosis," Physiological Measurement, Vol. 26, No. 5, 2005, pp. 865-874.

http://dx.doi.org/10.1088/0967-3334/26/5/022

[11] J. Allen and A. Murray, "Variability of Photoplethysmography Peripheral Pulse Measurements at the Ears, Thumbs, and Toes," IEE Proceedings on Science, Measurement and Technology, Vol. 147, No. 6, 2000, pp. 403407. http://dx.doi.org/10.1049/ip-smt:20000846

[12] P. A. Bonham, T. Kelechi, M. Mueller and J. Robison, "Are Toe Pressures Measured by a Portable Photo-Phlethysmograph Equivalent to Standard Laboratory Tests," Journal of Wound, Ostomy and Continence Nurses Society, Vol. 37, No. 5, 2010, pp. 475-486.

[13] J. Allen, K. Overbeck, A. F. Nath, A. Murray and G. Stansby, "A Prospective Comparison of Bilateral Photoplethysmography versus the Ankle-Brachial Pressure Index for Detecting and Quantifying Lower Limb Peripheral Arterial Disease," Journal of Vascular Surgery, Vol. 47, No. 4, 2008, pp. 794-802. http://dx.doi.org/10.1016/j.jvs.2007.11.057

[14] C.-H. Lin, "Assessment of Bilateral Photoplethysmography for Lower Limb Peripheral Vascular Occlusive Disease Using Color Relation Analysis Classifier," Computer Method and Program in Biomedicine, Vol. 103, No. 3, 2011, pp. 121-131.

[15] J. Allen, C. P. oates, T. A. Lees and A. Murray, "Photoplethysmography Detection of Lower Limb Peripheral Arterial Occlusive Disease: A Comparison of Pulse Timing, Amplitude and Shape Characteristics," Physiological Measurement, Vol. 26, No. 5, 2005, pp. 811-821. http://dx.doi.org/10.1088/0967-3334/26/5/018

[16] M. Fallahpour, D. Megias and M. Ghanbari, "Reversible 
and Highcapacity Data Hiding in Medical Images," IET Image Processing, Vol. 5, No. 2, 2011, pp. 190-197. http://dx.doi.org/10.1049/iet-ipr.2009.0226

[17] D. M. Vázquez, J. J. Rubio and J. Pacheco, "A Characterization Framework for Epileptic Signals," IET Image Processing, Vol. 6, No. 9, 2012, pp. 1227-1235. http://dx.doi.org/10.1049/iet-ipr.2012.0037

[18] H. Heidrich, R. Wenk and P. Hesse, "Frequency of Asymptomatic Peripheral Arterial Disease in Patients Entering the Department of General and Internal Medicine of a General Care Hospital," Vasa, Vol. 33, No. 2, 2004, pp. 63-67. http://dx.doi.org/10.1024/0301-1526.33.2.63

[19] I. Kauppinen, J. Kauppinen and P. Saarinen, "A Method for Long Extrapolation of Audio Signals," Journal of the Audio Engineering Society, Vol. 49, No. 12, 2001, pp. 1167-1180.

[20] A. Broadman, F. S. Schlindwein, A. P. Rocha and A. Leite, "A Study on the Optimum Order of Autoregressive Models for Heart Rate Variability," Physiological Measurement, Vol. 23, 2002, pp. 324-336.

[21] K. Roth, I. Kauppinen, P. A. A. Esquef and V. Valimaki, "Frequency Warped Burg's Method for AR-Modeling," IEEE Workshop on Applications of Signal Processing to Audio and Acoustics, 19-22 October 2003, pp. 5-8.

[22] C. Collomb, "Linear Prediction and Levinson-Durbin Algorithm," 2009.

http://www.emptyloop.com/technotes/A\%20tutorial\%20o $\mathrm{n} \%$ 20linear\%20prediction\%20and\%20Levinson-Durbin.p df

[23] N. Kannathal, U. Rajendra Acharya, P. Joseph and E. Y. $\mathrm{K}$. Ng, "Analysis of EEG Signals with and without Reflexology Using FFT and Auto Regressive Modeling Techniques," Chinese Journal of Medicine, Vol. 1, No. 1, 2006, pp. 12-20.

[24] M. Akay, J. L. Semmlow, W. Welkowitz, M. D. Bauer and J. B. Kostis, "Detection of Coronary Occlusions Using Autoregressive Modeling of Diastolic Heart Sounds," IEEE Transactions on Biomedical Engineering, Vol. 37, No. 4, 1990, pp. 366-373.

http://dx.doi.org/10.1109/10.52343

[25] S. B. Waluya and W. T. van Horssen, "On the Periodic Solutions of a Generalized Nonlinear Van der Pol Oscillator," Journal of Sound and Vibration, Vol. 268, No. 1, 2003, pp. 209-215.

http://dx.doi.org/10.1016/S0022-460X(03)00251-7
[26] Q. S. Bi, "Dynamical Analysis of Two Coupled Parametrically Excited Van der Pol Oscillators," International Journal of Nonlinear Mechanics, Vol. 39, No. 1, 2004, pp. 33-54. http://dx.doi.org/10.1016/S0020-7462(02)00126-9

[27] G. M. Mahmoud and A. A. M. Farghaly, "Chaos Control of Chaotic Limit Cycles of Real and Complex Van der Pol Oscillators," Chaos, Solitons and Fractals, Vol. 21, No. 4, 2004, pp. 915-924. http://dx.doi.org/10.1016/j.chaos.2003.12.039

[28] Y. Abbas, J. Ann and A. Merna, “A Multistage Adomian Decomposition Method for Solving the Autonomous Van Der Pol System," Australian Journal of Basic and Applied Sciences, Vol. 3, No. 4, 2009, pp. 4397-4407.

[29] N. S. Nise, “Control Systems Engineering," 4th Edition, John Wiley \& Sons, INC., 2004.

[30] Y. C. Du and C. H. Lin, "Adaptive Network-Based Fuzzy Inference System for Assessment of Lower Limb Peripheral Vascular Occlusive Disease," Journal of Medical System, Vol. 36, No. 1, 2012, pp. 301-310. http://dx.doi.org/10.1007/s10916-010-9476-1

[31] C. H. Lin, Y. F. Chen, Y. C. Du, J. X. Wu and T. S. Chen, "Chaos Synchronization Detector Combining Radial Basis Network for Estimation of Lower Limb Peripheral Vascular Occlusive Disease," Lecture Notes in Computer Science, Vol. 6165, 2010, pp. 126-136. http://dx.doi.org/10.1007/978-3-642-13923-9 13

[32] P. A. Bonham, T. Kelechi, M. Mueller and J. Robison, "Are Toe Pressures Measured by a Portable Photophlethysmograph Equivalent to Standard Laboratory Tests?" Journal of Wound Ostomy \& Continence Nursing, Vol. 37, No. 5, 2010, pp. 475-486. http://dx.doi.org/10.1097/WON.0b013e3181eda0c5

[33] P. Angelov, E. Lughofer and X. Zou, "Evolving Fuzzy Classifiers Using Different Model Architectures," Fuzzy Sets and Systems, Vol. 159, No. 23, 2008, pp. 3160-3182. http://dx.doi.org/10.1016/j.fss.2008.06.019

[34] J. J. Rubio, "SOFMLS: Online Self-Organizing Fuzzy Modified Least Squares Network," IEEE Transactions on Fuzzy Systems, Vol. 17, No. 6, 2009, pp. 1296-1309. http://dx.doi.org/10.1109/TFUZZ.2009.2029569

[35] C.-H. Lin and G.-W. Lin, "FPGA Implementation of Fractal Patterns Classifier for Multiple Cardiac Arrhythmias Detection," Journal of Biomedical Science and Engineering, Vol. 5, No. 3, 2012, pp. 120-132. 